\title{
Multi-Radio Mobile Device: Evaluation of Hybrid Node Between WiFi and LTE Networks
}

\author{
Pavel Masek, Krystof Zeman, Dalibor Uhlir, Jan Masek, Chris Bougiouklis, and Jiri Hosek
}

\begin{abstract}
With the ubiquitous wireless network coverage, Machine-Type Communications (MTC) is emerging to enable data transfers using devices / sensors without need for human interaction. In this paper we introduce a comprehensive simulation scenario for modeling and analysis heterogeneous MTC. We demonstrate the most expected scenario of MTC communication using the IEEE 802.11 standard for direct communication between sensors and for transmitting data between individual sensor and Machine-Type Communication Gateway (MTCG). The MTCG represents the hybrid node serving as a bridge between two heterogeneous networks (WiFi and LTE). Following the idea of hybrid node, two active interfaces must be implemented on this node together with mechanism for handling the incoming traffic (from WiFi network) to LTE network. As a simulation tool, the Network Simulator 3 (NS-3) with implemented LTE/EPC Network Simulator (LENA) framework was used. The major contribution of this paper therefore lies in the implementation of logic for interconnection of two heterogeneous networks in simulation environment NS-3.
\end{abstract}

Keywords-LTE, MTC Communication, MTCG, Network Simulator 3, WiFi.

\section{INTRODUCTION}

Machine-Type Communication (MTC) represents the way how to enable the connectivity between several (from tens to hundreds) nodes (sensors or actuators) without or with minimal human interaction e.g. Internet of Things (IoT) or smart power grids [1]. Following the information given in 2, 3, the amount of mobile data traffic is predicted to increase by around six times in the period 2014-2019. The data traffic is distinguished into two main categories: Human-to-Human $(\mathrm{H} 2 \mathrm{H})$ and Machineto-Machine (M2M) communication. In comparison with the traditional conception of data traffic represented by $\mathrm{H} 2 \mathrm{H}$ (services as voice, web streaming etc.), M2M comes with different requirements on a communication system 4

P. Masek, K. Zeman, D. Uhlir, and J. Hosek are with Department of Telecommunications, Brno University of Technology, Brno, Czech Republic (e-mails: xmasek12@phd.feec.vutbr.cz, xzeman43@stud.feec.vutbr.cz, xuhlir15@stud.feec.vutbr.cz, hosek@feec.vutbr.cz).

J. Masek is with Institute of Structural Mechanics, Brno University of Technology, Brno, Czech Republic (e-mail: masekj3@study.fce.vutbr.cz).

Ch. Bougiouklis is with Technological Educational Institute of Crete, Greece (e-mail: chrisbougiouklis@hotmail.com).

Manuscript received May 6, 2015; revised May 15, 2015. Research described in this paper was financed by the National Sustainability Program under grant LO1401 and by the project CZ.1.07/2.3.00/30.0005 of Brno University of Technology. For the research, infrastructure of the SIX Center was used. where the M2M applications should have minimal impact on existing $\mathrm{H} 2 \mathrm{H}$ services [5]. The key differences between both communication types are shown in Table II

The key idea of the M2M communication network is to connect a server with millions of devices deployed worldwide (interacting with other sensors, different environments and people). With the rapid development of cellular networks, M2M communication via the Long Term Evolution (LTE) network is expected to play a significant role in M2M scenarios. Today, the cellular networks represent the common data access to public network (Internet); as a consequence they are under pressure trying to handle unprecedented data flows from the side of mobile devices. The dramatic increase of transmitted data via cellular networks is a burning question for telecommunication operators with the limited resources of radio spectrum [6]. The complex scenario of M2M architecture is shown in Fig. 1

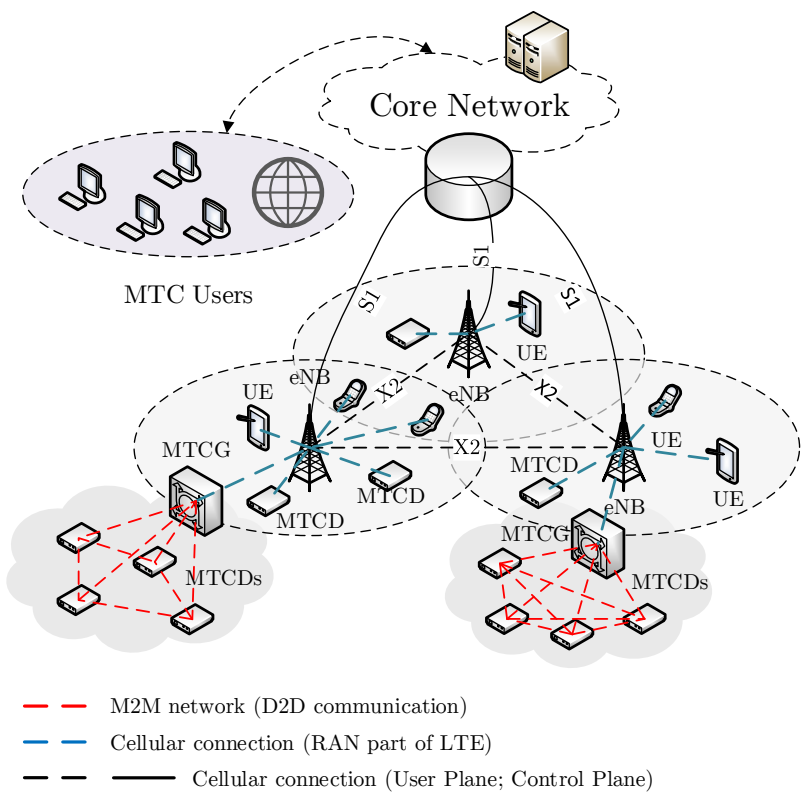

Fig. 1. LTE networks with M2M communications

The depicted architecture considers two different ways for managing connection of M2M devices to the core part of LTE network [8]:

- Cellular connectivity: connection through access network to core networks where each single device has its own Subscriber Identity Module (SIM) card for cellular connectivity. 
- M2M networks: M2M devices may create M2M area networks using short range technologies represented by the standards IEEE 802.15.6, IEEE 802.15.4(e), or IEEE 802.11. These M2M area networks can be then connected to the core networks via M2M gateways [9], [10], [11].

As a possible way how to deal with the overloading of Random Access Network (RAN) of LTE network, the offloading techniques can be used; offloading mechanisms refer to using alternative network infrastructure for transmitting data originally targeted for cellular network when this network becomes overloaded [7 $]^{1}$ Depending on delay (content delivery time) it is possible to divide offloading techniques into two categories: nondelayed offloading and delayed offloading [7.

In this paper we address the specific type of delayed offloading where the Machine Type Communication Gateway (MTCG) act as a hybrid node which interconnects two different networks (in case of this paper, WiFi and LTE network are considered as heterogeneous networks). The attention is also paid to the implementation of Quality of Service (QoS) for $\mathrm{H} 2 \mathrm{H}$ and M2M communication where the high prioritized traffic is represented by the $\mathrm{H} 2 \mathrm{H}$ communication (e.g. Voice over IP (VoIP)); QoS is implemented on MTCG node. Furthermore, QoS requirements of M2M services depend on the MTC service features: group-based communication, mobility, timecontrolled/time-tolerant, amount of transmitted data, power consumption 12 .

${ }^{1}$ Current visions from analytical claim that by 2019, 54 percent of total mobile data traffic will be offloaded over WiFi networks.
We performed extensive simulations to evaluate the role of MTCG node in LTE architecture with M2M communication. For modeling WiFi and LTE networks, data traffic and logic of MTCG node, the simulation tool Network Simulator 3 (NS-3) [13] with the framework LTE/EPC Network Simulator (LENA) [14] was used.

The rest of the paper is organized as follows. Section II presents the description of MTCD-Related communications in LTE network. Section III deals with the selected simulation environment NS-3 together with the LENA framework. In section IV the description of created simulation scenario is given. Section $\mathrm{V}$ presents the obtained results and finally, in section $\overline{V I}$ we draw a conclusion with our future plans in this research area.

\section{LTE NETWORK AND M2M COMmunicATION}

The Current RAN for LTE network consists of eNodeB (eNB) that provides the user plane and control plane protocol stack for the User Equipment (UE). LTE represents the fully distributed radio access network architecture, where the eNB can be interconnected with other eNBs by the X2 interface. The eNBs are then connected to the core part of LTE network through the S1 interface, see Fig. 1. Each eNB includes layers below that implement the functionality of user plane, header compression and encryption [12]:

- PHY: Physical layer,

- MAC: Medium Access Control layer,

- RLC: Radio Link Control layer,

- PDCP: Packet Data Control Protocol layer.
Machine-to-Machine (M2M)

\begin{tabular}{|c|c|c|}
\hline Traffic Direction & $\begin{array}{l}\text { Uplink data; data received from sensors. For a specific } \\
\text { type of the applications, the symmetric uplink and } \\
\text { downlink is needed to fulfill the requirements for the } \\
\text { dynamic interaction. }\end{array}$ & $\begin{array}{l}\text { Downlink data; although during last few years the } \\
\text { amount of uploaded data is growing fast, in case of } \\
\mathrm{H} 2 \mathrm{H} \text {, download still represents the main part of data } \\
\text { traffic. }\end{array}$ \\
\hline Message Size & $\begin{array}{l}\text { Size of data from sensors is in general very small (e.g. } \\
\text { data size of Wireless M-BUS data unit is usually max. } \\
50 \mathrm{~B}) \text {. }\end{array}$ & $\begin{array}{l}\text { Using multimedia and realtime applications, the size of } \\
\text { data units is several times higher in comparison with } \\
\text { the M2M. }\end{array}$ \\
\hline $\begin{array}{l}\text { Access } \\
\text { Delay }\end{array}$ & $\begin{array}{l}\text { For the dynamic interaction between sensors and ac- } \\
\text { tuators, delays should be very short. }\end{array}$ & $\begin{array}{l}\text { In case of } \mathrm{H} 2 \mathrm{H} \text { communication, longer access delays } \\
\text { are usually tolerated. }\end{array}$ \\
\hline $\begin{array}{l}\text { Transmission Peri- } \\
\text { odicity }\end{array}$ & $\begin{array}{l}\text { The range of transmitting period can be from units } \\
\text { of seconds (e.g. alarm systems) up to tens of minutes } \\
\text { (e.g. energy meters). }\end{array}$ & $\begin{array}{l}\text { Nature of human based traffic is mostly random and } \\
\text { bursty. Therefore, the often sending of control infor- } \\
\text { mation is required (to ensure QoS). }\end{array}$ \\
\hline Mobility & $\begin{array}{l}\text { For the main group of sensors, mobility does not } \\
\text { represent a big issue (sensors are mostly located at the } \\
\text { stable position). }\end{array}$ & $\begin{array}{l}\text { For humans, mobility management represents a key } \\
\text { requirement for ensuring seamless connectivity and } \\
\text { roaming. }\end{array}$ \\
\hline $\begin{array}{l}\text { Data } \\
\text { Importance }\end{array}$ & $\begin{array}{l}\text { Some of the } \mathrm{M} 2 \mathrm{M} \text { sensors can transmit critical data } \\
\text { (e.g. status of alarm system). Following this fact, M } 2 \mathrm{M} \\
\text { data requires high priority. }\end{array}$ & $\begin{array}{l}\text { There are no big differences between users. The dif- } \\
\text { ferences could be found between the applications for } \\
\text { individual users (with respect to QoS and QoE). }\end{array}$ \\
\hline Amount of devices & $\begin{array}{l}\text { Hundreds or thousands of devices connected via one } \\
\text { access point to the network. }\end{array}$ & $\begin{array}{l}\text { Typically tens of devices which are connected via } \\
\text { access point to the network. }\end{array}$ \\
\hline $\begin{array}{l}\text { Lifetime; Energy } \\
\text { Efficiency }\end{array}$ & $\begin{array}{l}\text { Using specific energy profiles, devices are able to oper- } \\
\text { ate for years of decades without human maintenance. }\end{array}$ & $\begin{array}{l}\text { In case of devices used by humans, it is common to } \\
\text { recharge batteries in a daily manner (smartphones, } \\
\text { laptops). }\end{array}$ \\
\hline
\end{tabular}

TABLE I

DifFERENCES Between H2H AND M2M COMMUNiCATion 8 
Following the fact that the current $3 \mathrm{G}$ cellular networks are designed only for $\mathrm{H} 2 \mathrm{H}$ communications, the introduction of M2M communications introduce the new requirements on LTE networks; the network architecture needs to be improved to fulfill M2M services without sacrificing the current $\mathrm{H} 2 \mathrm{H}$ applications.

In this section, the attention will be given to a description of types of M2M communication (especially a description of connection of the MTCD and MTCG nodes to LTE network will be described).

\section{A. Machine Type Communication}

To enable M2M communication in cellular networks (3G / 4G), the two new types of nodes Machine Type Communication Devices (MTCD) and Machine Type Communication Gateway $(M T C G)$ were introduced. The MTCD represents the UE which is supposed to work as a sensor which communicates through the cellular network with the remote MTC node (e.g. database server) or (and) with other MTCDs in range. As was proven in [15], the high number of MTCDs connected at the same time to one eNB may cause overloading of this network entity. Therefore, the cellular network requires an MTCG node to facilitate communications among a great number of MTCDs. The MTCG will enable the intelligent way how to manage power consumption of MTCDs and provide an efficient path for communication between MTCDs without the need of connection to the LTE network. Three different M2M communication methods were introduced during last few years, see Fig. 2 12. These methods are described (in the text) below.

1) Direct Transmission Between MTCD and eNB: The first method is similar to the classic UE where the MTCD is able to establish the direct connection to the eNB; therefore similarities between eNB-to-UE and eNBto-MTCD exist. On the other hand, the MTCDs are represented in a large amount of sensors / UEs; in certain time period, intensive competition for radio resources may occur. Therefore, the additional efforts have to be covered by the telecommunication operators to solve the problems, when the large number of MTCDs communicate with the eNB directly [12.

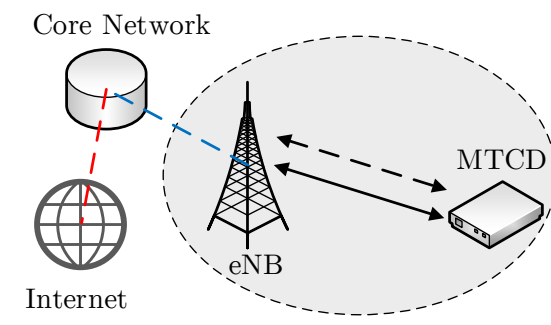

a)

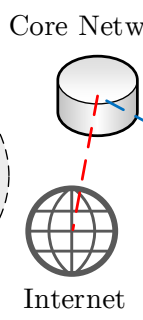

Internet

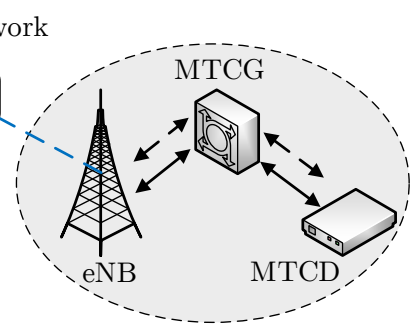

b)
2) Multihop Transmission using MTCG: With respect to mitigate or eliminate negative effect of M2M communication on $\mathrm{H} 2 \mathrm{H}$ communication in cellular network, the MTCG node can be deployed as a hybrid node, where all MTCDs are connected to the eNB indirectly using the MTCG node as a gateway. The eNB-to-MTCG connection is based on the 3GPP (Third Generation Partnership Project) LTE specifications. The MTCG-to-MTCDs and MTCD-to-MTCD communications can be established via $3 \mathrm{GPP}$ LTE specifications or via the non-3GPP communication technologies such as IEEE 802.11, IEEE 802.15.x [12, [16].

3) P2P Transmission Between MTCDs: An MTCD may communicate in local area with other MTCDs and with the eNB. Compared to other non-3GPP local connectivity solutions (IEEE 802.11, IEEE 802.15.x), direct communication between MTCDs is done by cellular network which can broadcast data within a much wider coverage area. For service discovery, the MTCDs do not have to scan all the time for the available access point (APs) as in the case of standard IEEE 802.11 [12, [16, [17].

\section{LENA FRAMEWORK IN NS-3}

During the last years, several network simulation platforms have been developed as a tool available for networking research: OPNET Modeler [19, OMNET++ [18], NS-2 13], NS-3 [13. Based on the fact that this paper deals with the M2M communication in LTE network, the simulation environment NS-3 together with the LENA framework 21 were used. In our work, we used NS-3 in version 3.21 together with the LENA framework in version 8. Using the LENA inside NS-3 provides for us the way for design and performance evaluation of Heterogeneous Networks (HetNets). Fig. 3 shows the implementation of the end-to-end LTE-EPC data plane protocol stack of LENA framework. The biggest change in comparison with the standard implementation of data plane protocol stack of LTE is the merge of the Serving Gateway (SGW) and PDN Gateway (PGW) functionality within one single $(\mathrm{SGW}) /(\mathrm{PGW})$ node in NS-3. This change causes that there is no need to have S5 and S8 interfaces which are specified by 3GPP. The S1-U protocol stack and the LTE radio protocol stack specified by $3 \mathrm{GPP}$, are also described in Fig. 3 


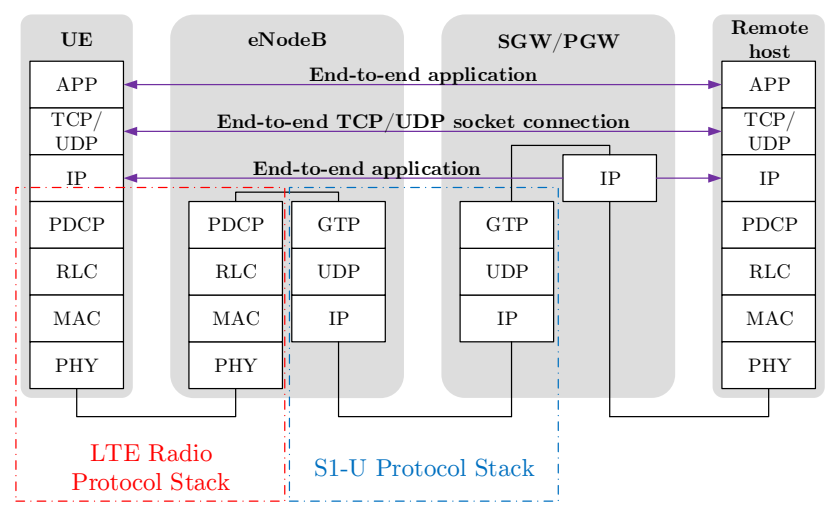

Fig. 3. LTE-ECP data plane protocol stack in LENA framework

\section{Model of M2M Communication in LTE NETWORK}

As described in Section II] the created scenario includes the MTCD nodes together with the MTCG node which enables the interconnection between the local network and the public network (represented by the remote host which is accessible through the LTE network). The local side of the implemented scenario is represented by sensors / UEs using IEEE 802.11g, IEEE 802.11ah 23, 24 and Wireless M-BUS 22] which represent the most preferred technologies for M2M. The data is sent through the hybrid node (MTCG) to a remote host which is accessible via the LTE network. The overall structure of the created scenario is depicted in Fig. 4

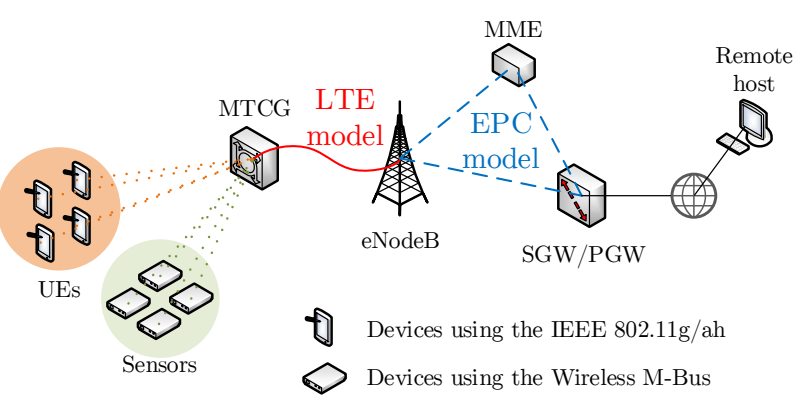

Fig. 4. M2M communication scenario in NS-3

\section{A. Parameters of Simulation Scenario}

The key parameters of created simulation model are shown in Table II (a list of the parameters of created LTE network using the LENA framework).

UEs were created as wireless nodes using the IEEE 802.11 g and IEEE 802.11 ah for connection to MTCG node. The sensors implemented the Wireless M-BUS communication protocol $(868 \mathrm{MHz})$ where the sensors were set to Mode T1 (one-directional communication) and MTCG was set to the Mode T2 (bi-directional communication).
TABLE II

PARAmeters OF LTE NETWORK IN NS-3

\begin{tabular}{ll} 
Parameter & Setting \\
\hline Cell Layout & 1 eNodeB, 1 sector \\
\hline Duplex Format & LTE-FDD \\
\hline Maximum transmit power & $30 \mathrm{dBm}$ \\
\hline System Bandwith & $3 \mathrm{MHz}(\sim 15 \mathrm{PRBs})$ \\
\hline Scheduler & Pf Df Mac Scheduler \\
\hline Path loss model & Friis Spectrum Propagation \\
\hline Direction & Loss Model \\
\hline eNB antenna model & Download \\
\hline Frequency Reuse Factor & Isotropic Antenna Model \\
\hline
\end{tabular}

\section{B. IP Address Scheme}

The address scheme for two groups of nodes is depicted in Fig. 5. For the WiFi nodes (IEEE 802.11 g/ah) the address space 10.3.0.0 with prefix 24 was used. In case of Wireless M-BUS nodes, unique addressing scheme is implemented following the 25]. The address of each WMBUS node is represented by the serial number of sensor.

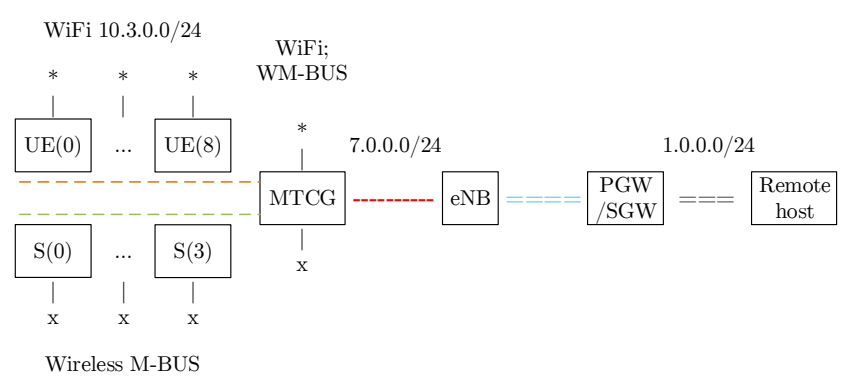

Fig. 5. Address Scheme of created model

The transmission of data from sensors is performed as a broadcast communication when only the MTCG node (in T2 mode) can receive the information from sensors. Data from MTCG node goes through the core part of LTE network (7.0.0.0 / 24) to the destination node (remote host; 1.0.0.0 / 24).

\section{Parameters of Data Traffic}

Data traffic is generated independently by a group of UEs $(\mathrm{H} 2 \mathrm{H})$ and sensors (M2M). Data traffic from UEs represents the voice service defined as follows [26]: UDP transport protocol; packet size $160 \mathrm{~B}$; Maximal Transfer Unit (MTU) 1500 B. Traffic from sensors was generated with these attributes: WM-BUS communication protocol (do not follow the TCP/IP reference model), packet size $50 \mathrm{~B}$, transmission interval 30 seconds. Both groups of devices (UEs and sensors) generate traffic during the whole simulation; simulation time was set to 10 minutes.

\section{In-DePth Results Discussion}

From the implementation point of view, the two active interfaces on one node in NS-3 represent a challenging task. This task is going to be more complex when one 
of the implemented interface does not follow the requirements given by TCP/IP reference model; this is an example of Wireless M-BUS, which was implemented from the scratch in NS-3 as a specific representative of M2M data traffic. Merging the data traffic (the part of static routing for WiFi nodes which have been configured with the static route to the MTCG node) is briefly described in a part of the source code below.

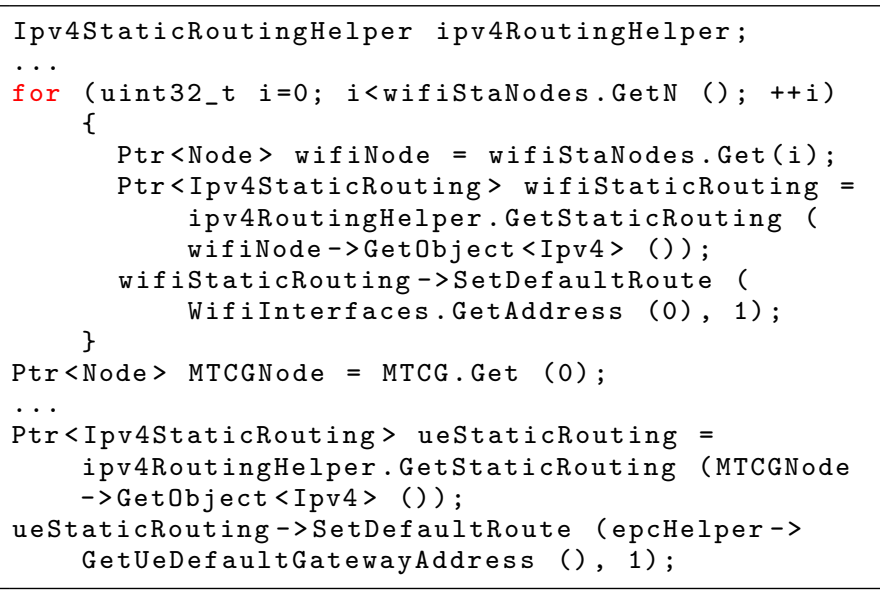

The example of one data frame received from sensors using the Wireless M-BUS on MTCG is shown below 2 .

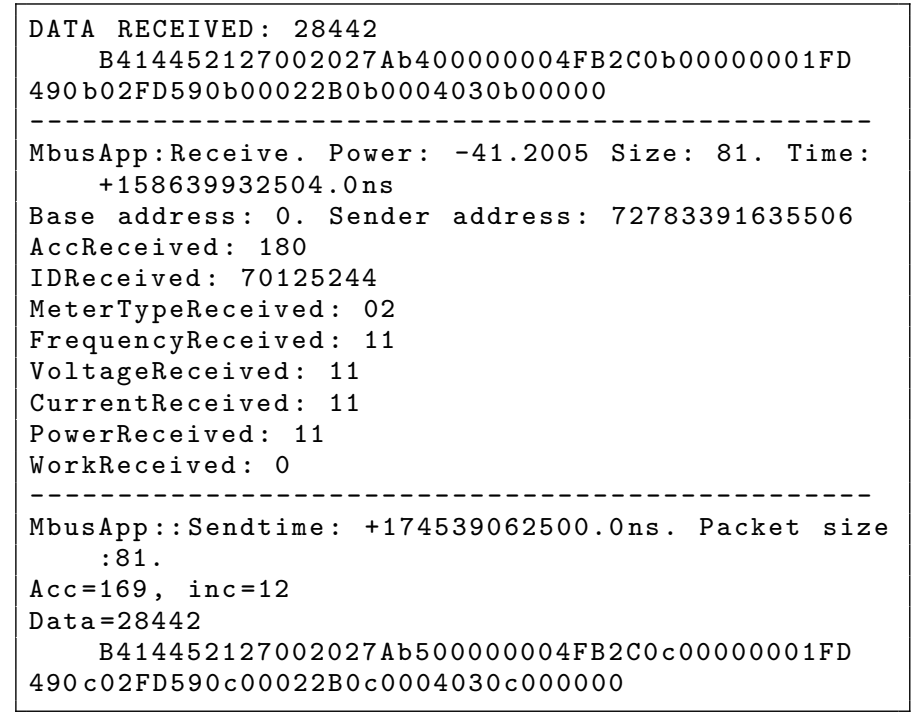

\section{A. Analysis of Data Traffic}

To evaluate the correct behavior of created traffic from UEs and sensors, the trace files were created during the simulation in a compatible format for network protocol analyzer Wireshark [31. As depicted in Fig. 3, the UDP is used as a transport protocol in local network. On the other hand, in LTE network the data is encapsulated via the GPRS Tunneling Protocol (GTP) 32 which is used over S1-U, X2, S4, S5 and S8 interfaces of the Evolved Packet System (EPS); note that the S5 and S8 interfaces are not implemented in LENA framework yet. GTP is an important IP/UDP based protocol used in Global

${ }^{2}$ The specifications of sensors manufacturers Bonega 27, WepTech
28 , Pikkerton 29, and ZPA 30 were implemented and evaluated.
System for Mobile Communications (GSM), Universal Mobile Telecommunication System (UMTS) and LTE core networks.

The correct handling with the data traffic is depicted for the UE(0) in Fig. 6 and Fig. 7

\begin{tabular}{|c|c|c|c|c|c|}
\hline No. & Time & Source & Destination & \multicolumn{2}{|c|}{ Protocol Length } \\
\hline & 10.000000 & $10.3 \cdot 0.1$ & 1.0 .0 .2 & UDP & 1500 \\
\hline & 20.112000 & 10.3 .0 .1 & 1.0 .0 .2 & UDP & 1500 \\
\hline & 30.230000 & 10.3 .0 .1 & 1.0 .0 .2 & UDP & 1500 \\
\hline & 40.347000 & 10.3 .0 .1 & 1.0 .0 .2 & UDP & 1500 \\
\hline & 50.465000 & 10.3 .0 .1 & 1.0 .0 .2 & UDP & 1500 \\
\hline \multicolumn{6}{|c|}{ Frame 1: 1500 bytes on wire ( 12000 bits), 1500 bytes captured ( 12000 bits) } \\
\hline \multicolumn{6}{|c|}{$\begin{array}{l}\text { Point-to-Point Protocol } \\
\text { Internet Protocol Version 4, Src: } 10.3 .0 .1(10.3 .0 .1) \text {, Dst: } 1.0 .0 .2(1.0 .0 .2) \\
\text { User Datagram Protocol, Src Port: } 49153 \text { (49153), Dst Port: } 8 \text { (8) } \\
\text { Data (1470 bytes) }\end{array}$} \\
\hline
\end{tabular}

Fig. 6. Captured data traffic in local network; UDP protocol

\begin{tabular}{|c|c|c|c|c|c|}
\hline No. & Time & Source & Destination & Protocol & ngth \\
\hline & 10.000000 & 10.3 .0 .1 & 1.0 .0 .2 & GTP $\langle$ UDP $\rangle$ & 1540 \\
\hline & 20.112000 & 10.3 .0 .1 & 1.0 .0 .2 & GTP $\langle$ UDP $\rangle$ & 1540 \\
\hline & 30.230000 & 10.3 .0 .1 & $1 . \theta . \theta .2$ & GTP $\langle$ UDP $\rangle$ & 1540 \\
\hline & 40.347000 & 10.3 .0 .1 & 1.0 .0 .2 & GTP $\langle$ UDP $\rangle$ & 1540 \\
\hline & 50.465000 & 10.3 .0 .1 & $1.0 . \theta .2$ & GTP $\langle$ UDP $\rangle$ & 1540 \\
\hline \multirow{2}{*}{\multicolumn{6}{|c|}{ 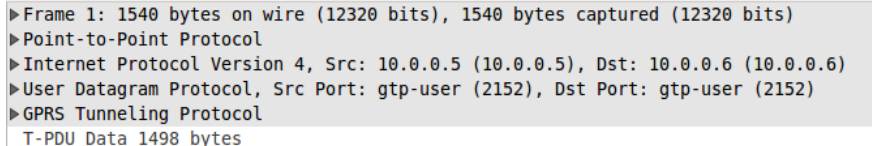 }} \\
\hline & & & & & \\
\hline \multicolumn{6}{|c|}{$\begin{array}{l}\text { DInternet Protocol Version 4, Src: } 10.3 .0 .1(10.3 .0 .1) \text {, Dst: } 1.0 .0 .2(1.0 .0 .2) \\
\text { User Datagram Protocol, Src Port: } 49153(49153), \text { Dst Port: } 8(8) \\
\text { Data (1470 bytes) }\end{array}$} \\
\hline
\end{tabular}

Fig. 7. Captured data traffic in LTE network; GTP protocol

\section{B. Enabling QoS for H2H Traffic in Created Model}

The support of QoS for VoIP data traffic (originated from $\mathrm{WiFi}$ nodes (UEs)) was implemented on MTCG node. The situation with and without the implemented QoS features is depicted in Fig. 8.

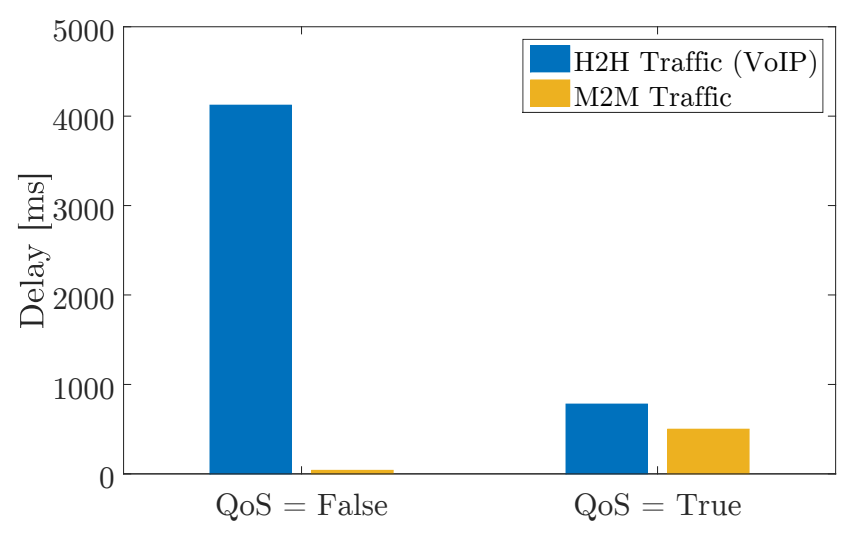

Fig. 8. Implemented QoS features on MTCG node

The values of delay were originally for $\mathrm{H} 2 \mathrm{H}$ traffic (VoIP) $4123 \mathrm{~ms}$ and $40 \mathrm{~ms}$ for M2M traffic. It is clearly visible that without the implemented QoS, the VoIP services can not be used with respect to fulfill users expectation. Therefore, the QoS was implemented on MTCG node and the delay decreased to $780 \mathrm{~ms}$ (this means an improvement of $81.08 \%$ in comparison with the original delay for VoIP). 


\section{CONCLUSION}

M2M communications represent an emerging technology which illustrates the principles of the IoT. Therefore, it has gained an increasing attention in LTE / LTE-A cellular network design. In this paper, we give the overview of the required network architectural improvements with the description of the various transmission schemes/types for MTCDs. We chose the multihop transmission from described transmission schemes, see Fig. 2 This type can be represented by the MTCG node which acts as a hybrid node between several heterogeneous networks. In this paper we implemented three types of networks: WiFi, Wireless M-BUS and LTE. Between these networks the MTCG node was deployed in a role of the bridge where the incoming data traffic is routed towards the destination node (e.g. a remote server located in Internet).

The implementation was done using the simulation environment NS-3 with the LENA framework, see section III The simulation results, see section V] confirm the correct handling of data traffic with respect to meet the QoS and QoE requirements for the $\mathrm{H} 2 \mathrm{H}$ traffic in case when the M2M services are deployed in parallel with the $\mathrm{H} 2 \mathrm{H}$. Although we have achieved an improvement of delay of $84 \%$ (from $4123 \mathrm{~ms}$ to $780 \mathrm{~ms}$ ) for VoIP services, it is evident that further investigation of aggregation scheme on MTCG node is still needed.

\section{ACKNOWLEDGMENT}

The described research was supported by the National Sustainability Program under grant LO1401 and by the project CZ.1.07/2.3.00/30.0005 of Brno University of Technology. For the research, infrastructure of the SIX Center was used.

\section{REFERENCES}

[1] F. Ghavimi and Ch. Hsiao-Hwa, M2M Communications in 3GPP LTE/LTE-A Networks: Architectures, Service Requirements, Challenges and Applications, Communications Surveys \& Tutorials, IEEE , vol.PP, no.99, pp.1,1 doi: 10.1109/COMST.2014.2361626

[2] Cisco, Visual Networking Index: Global Mobile Data Traffic Forecast Update 20142019 White Paper. Cisco [online]. Available from: http://bit.ly/1b13ryX

[3] Ericsson, Annual Report 2014. [online]. Available from: http://bit.ly/1Gc9lId

[4] 3GPP TS 22.368 v1.0, Service requirements for Machine-Type Communications (MTC) Stage 1 (Release 10), Mar. 2010.

[5] 3GPP TR 23.888 v0.5.1, System Improvements for MachineType Communications (Release 10), July 2010.

[6] D.H. Hagos and R. Kapitza, Study on performance-centric offload strategies for LTE networks, Wireless and Mobile Networking Conference (WMNC), 2013 6th Joint IFIP , p.1-10, 2325 April 2013. DOI: 10.1109/WMNC.2013.6548999

[7] F. Rebecchi, D. Amorim, V. Conan, A. Passarella, R. Bruno and M. Conti, Data Offloading Techniques in Cellular Networks: A Survey, IEEE Communications Surveys \& Tutorials, vol.PP, no.99,.

[8] A. Laya, L. Alonso and J. Alonso-Zarate, Is the Random Access Channel of LTE and LTE-A Suitable for M2M Communications? A Survey of Alternatives, Communications Surveys \& Tutorials, IEEE , vol.16, no.1, pp.4,16, First Quarter 2014 doi: 10.1109/SURV.2013.111313.00244

[9] P. Masek, J. Hosek, D. Kovac and F. Kropfl, M2M Gateway: The Centrepiece of Future Home. In 2014 6th International Congress on Ultra Modern Telecommunications and Control Systems and Workshops (ICUMT). St. Petersburg, Russia: 2014. s. 286-293. ISBN: 978-1-4799-5290- 8 .
[10] J. Hosek, P. Masek, D. Kovac, M. Ries and F. Kropfl, IP Home Gateway as Universal Multi- Purpose Enabler for Smart Home Services. Elektrotechnik und Informationstechnik ÃÜVE - Verbandszeitschrift, 2014, roÄD. 131, ÄD. 5, s. 1-6. ISSN: 0932- 383X.

[11] J. Hosek, P. Masek, D. Kovac, M. Ries and F. Kropfl, Universal Smart Energy Communication Platform. In 2014 International Conference on Intelligent Green Building and Smart Grid (IGBSG). 1. Taipei, Taiwan: IEEE, 2014. s. 1-4. ISBN: 9781467361217.

[12] K. Zheng, H. Fanglong, W. Wenbo, W. Xiang and M. Dohler, Radio resource allocation in LTE-advanced cellular networks with M2M communications, Communications Magazine, IEEE , vol.50, no.7, pp.184,192, July 2012 doi: 10.1109/MCOM.2012.6231296

[13] Network Simulator 3: Discrete-event network simulator. NSNAM [online]. Available from: www.nsnam.org

[14] LENA: LTE-EPC Network simulAtor. CTTC [online]. Available from: http://networks.cttc.es/mobile-networks/softwaretools/lena/

[15] P. Masek, J. Hosek and M. Dubrava, Influence of M2M Communication on LTE Networks. In Sbornik prispevku studentske konference Zvule 2014. 1. 2014. s. 53-56. ISBN: 978-80-214-50059 .

[16] P. Marsch et al., Future mobile communication networks: Challenges in the design and operation, IEEE Veh. Technol. Mag. vol. 7, no. 1, pp. 16âĂŞ23, Mar. 2012.

[17] A. Aijaz, H. Aghvami and M. Amani, A survey on mobile data offloading: technical and business perspectives, Wireless Communications, IEEE, vol.20, no.2, pp.104,112, April 2013 doi: 10.1109/MWC.2013.6507401

[18] OMNeT++: Discrete Event Simulator. [online]. Available from: www.omnetpp.org

[19] Riverbed Modeler: Network Performance Management. Riverbed [online]. Available from: http://bit.ly/1Mpu9OC

[20] Network Simulator 2: Discrete event simulator targeted at networking research. [online]. Available from: http://www.isi.edu/nsnam/ns/

[21] LENA: LTE-EPC Network simulAtor. CTTC [online]. Available from: http://networks.cttc.es/mobile-networks/softwaretools/lena/

[22] S. Spinsante, M. Pizzichini, M. Mencarelli, S. Squartini and E. Gambi, Evaluation of the Wireless M-Bus standard for future smart water grids, in Wireless Communications and Mobile Computing Conference (IWCMC), 2013 9th International, 2013, pp. 1382âĂŞ1387.

[23] Y. Zhou, H. Wang, S. Zheng, Z.Z. Lei, Advances in IEEE 802.11ah standardization for machine-type communications in sub-1GHz WLAN, Communications Workshops (ICC), 2013 IEEE International Conference on , vol., no., pp.1269,1273, 9-13 June 2013 doi: 10.1109/ICCW.2013.6649432

[24] T. Adame, A. Bel, B. Bellalta, J. Barcelo, M. Oliver, IEEE 802.11AH: the WiFi approach for M2M communications, Wireless Communications, IEEE , vol.21, no.6, pp.144,152, December 2014 doi: 10.1109/MWC.2014.7000982

[25] EN 13757-4. Communication systems for meters and remote reading of meters - Part 4: Wireless meter readout (Radio Meter reading for operation in the 868-870 MHz SRD band). Brusel: European Committee for Standardization, 2003. Available from: http://oldfjarrvarme.unc.se/download/1309/fj

[26] Cisco. Voice Over IP - Per Call Bandwidth Consumption. Cisco [online]. Available from: http://www.cisco.com/c/en/us/support/docs/voice/voicequality/7934-bwidth-consume.html

[27] Bonega: Water-Meters and Accessories. Available from: http://bit.ly/1F0LQo1

[28] WepTech: Wireless Technology. Available from: https://www.weptech.de/

[29] Pikkerton: Wireless M-BUS devices. Available from: http://www.pikkerton.com/

[30] ZPA Smart Energy: Going the Smarter Way. Available from: http://www.zpa.cz/

[31] Wireshark: Network protocol analyzer. Available from: https://www.wireshark.org/

[32] 3GPP TS 29.281: General Packet Radio System (GPRS) Tunnelling Protocol User Plane (GTPv1-U). 